\title{
The Existence of Multiple Solutions for Nonhomogeneous Kirchhoff Type Equations in $\mathbb{R}^{3}$
}

\author{
Qi Zhang and Xiaoli Zhu \\ School of Mathematical Sciences, Shanxi University, Taiyuan 030006, China \\ Correspondence should be addressed to Qi Zhang; zhangqi@sxu.edu.cn \\ Received 6 September 2013; Accepted 17 November 2013 \\ Academic Editor: Csaba Varga
}

Copyright (c) 2013 Q. Zhang and X. Zhu. This is an open access article distributed under the Creative Commons Attribution License, which permits unrestricted use, distribution, and reproduction in any medium, provided the original work is properly cited.

We are concerned with the existence of multiple solutions to the nonhomogeneous Kirchhoff type equation $-\left(a+b \int_{\mathbb{R}^{3}}|\nabla u|^{2}\right) \Delta u+u=$ $|u|^{p-1} u+h(x)$ in $\mathbb{R}^{3}$, where $a, b$ are positive constants, $p \in(1,5), 0 \leqslant h(x)=h(|x|) \in C^{1}\left(\mathbb{R}^{3}\right) \cap L^{2}\left(\mathbb{R}^{3}\right)$, we can find a constant $m_{p}>0$ such that for all $p \in(1,5)$ the equation has at least two radial solutions provided $|h|_{2}<m_{p}$.

\section{Introduction and Main Result}

In this paper, we consider the existence of multiple solutions to the following nonhomogeneous Kirchhoff type equation:

$$
-\left(a+b \int_{\mathbb{R}^{3}}|\nabla u|^{2}\right) \Delta u+u=|u|^{p-1} u+h(x) \quad \text { in } \mathbb{R}^{3}
$$

where $a, b$ are positive constants and $p \in(1,5) \cdot h \in C^{1}\left(\mathbb{R}^{3}\right) \cap$ $L^{2}\left(\mathbb{R}^{3}\right)$ satisfies the following conditions:

$\left(\mathrm{h}_{1}\right) \quad 0 \leqslant h(x)=h(|x|) \in L^{2}\left(\mathbb{R}^{3}\right)$ and $|h|_{2} \leqslant m_{p}$, where

$$
m_{p}=\frac{p-1}{2 p \gamma_{2}}\left(\frac{p+1}{2 p \gamma_{p+1}^{p+1}}\right)^{1 /(p-1)}
$$

$\gamma_{s}$ is the embedding coefficient of $H^{1}\left(\mathbb{R}^{3}\right) \hookrightarrow$ $L^{s}\left(\mathbb{R}^{3}\right)$ and $s \in[2,6]$;

$\left(\mathrm{h}_{2}\right)(\nabla h(x), x) \in L^{2}\left(\mathbb{R}^{3}\right)$, where $(\cdot, \cdot)$ denotes the usual inner product in $\mathbb{R}^{3}$.

Recently, there have been many references about the existence of nontrivial solutions to the following Kirchhoff type equation by using variational method [1-5]:

$$
-\left(a+b \int_{\mathbb{R}^{3}}|\nabla u|^{2}\right) \Delta u+V(x) u=f(x, u) \quad \text { in } \mathbb{R}^{N},
$$

where $a, b$ are positive constants. $V: \mathbb{R}^{N} \rightarrow \mathbb{R}, f \in$ $C\left(\mathbb{R}^{N} \times \mathbb{R}, \mathbb{R}\right), N=1,2,3$. A main tool to deal with problem (3) is the mountain pass theorem. For this purpose, one usually assumes that $f(x, t)$ is subcritical, superlinear at the origin, and either 4-superlinear at infinity or satisfies the following global Ambrosetti-Rabinowitz type condition (AR in short):

(AR) there exists $\mu>4$ such that $0<\mu F(x, t)=$ $\int_{0}^{t} f\left(x, s \leqslant t f(x, t)\right.$ for all $x \in \mathbb{R}^{N}$ and $t \in \mathbb{R}$. Under the above assumptions, the mountain pass geometry structure and the boundedness of PalaisSmale sequence or Cerami sequence can be obtained.

For example, in [5], when $f$ satisfies above assumptions and the potential $V$ satisfies the following conditions:

(V) $V \in C\left(\mathbb{R}^{N}, \mathbb{R}\right), \inf _{\mathbb{R}^{N}} V>0$ and for each $M>0$, meas $\left\{x \in \mathbb{R}^{N}: V(x) \leqslant M\right\}<\infty$, where meas denotes the Lebesgue measure,

which ensure the compact imbedding of $E=\left\{u \in H^{1}\left(\mathbb{R}^{N}\right)\right.$ : $\left.\int_{\mathbb{R}^{N}} V u^{2}<\infty\right\} \hookrightarrow L^{q}\left(\mathbb{R}^{N}\right), q \in\left[2,2^{*}\right)$, the author obtained the existence of a nontrivial solution to problem (3).

The existence of infinitely many solutions was considered in $[2,3]$ respectively, by the fountain theorem and a variant version of the fountain theorem, where $f$ is odd on $t \in$ $\mathbb{R}$ and is also subcritical, superlinear at the origin, and either 4-superlinear at infinity or satisfies AR condition or some 
conditions weaker than AR condition. In [2], $N=2,3, V \equiv$ 1 and in [3], $N=3, V \in L_{\text {loc }}^{\infty}\left(\mathbb{R}^{3}\right)$ satisfies the condition $(\mathrm{V})$.

The existence of ground state solutions to problem (3) was also considered in $[1,4]$. In [1], the authors studied (3) under the conditions: $N=3$, a positive potential satisfies $V_{\infty}=\liminf _{|x| \rightarrow \infty} V(x)>V_{0}=\inf _{x \in \mathbb{R}^{N}}>$ $0 \cdot f(x, t)=f(t) \in C^{1}(\mathbb{R}, \mathbb{R})$ satisfies $(\mathrm{AR}), \lim _{t \rightarrow 0}$ $\left(f(t) /|t|^{3}\right)=0, \lim _{t \rightarrow \infty}\left(f(t) /|t|^{q}\right)=0$ for some $q \epsilon$ $(3,5)$ and $f(t) / t^{3}$ increases for all $t>0$. They obtained a positive ground state solution by using the Nehari manifold.

Under the same condition of $V$ in [1], the authors in [4] discussed the existence of multiple ground state solutions, where $f(x, t)=\lambda f(t)+|t|^{4} t$, which contains a critical growth term.

Recently, in [6], the authors studied the existence of a positive solution for the following Kirchhoff equation:

$$
\left(a+\lambda \int_{\mathbb{R}^{3}}\left[|\nabla u|^{2}+b u^{2}\right]\right)(-\Delta u+b u)=f(u) \text { in } \mathbb{R}^{3}
$$

where $N \geqslant 3, a, b>0, f$ is subcritical, superlinear at the origin and infinity. In order to construct the mountain pass geometry structure and obtain the bounded PS sequence, they combined a truncation argument with a monotonicity trick introduced by Jeanjean [7], and obtained that there exists $\lambda_{0}>0$ such that problem (4) has at least one positive solution for $\lambda \in\left(0, \lambda_{0}\right)$.

Motivated by the aformentioned references, we consider the existence of multiple solutions to the nonhomogeneous Kirchhoff equation (1), where $p \in(1,5)$. By using the variational method, we obtain that the problem has at least two positive radial solutions. Under proper assumptions on $h$, the problem has a local minimum around the origin with negative energy by Ekeland variational principle. Note that the term $|u|^{p-1} u$ is neither 4-superlinear nor satisfies AR condition for $p \in(1,3]$. In order to obtain the bounded PS sequence, we also use the indirect method in [7]. Meanwhile, for $w \in H^{1}\left(\mathbb{R}^{3}\right)$, we take a transform of $w_{t}(\cdot)=t w\left(t^{-2} \cdot\right)$ to construct the mountain pass geometry structure. Finally, the combination of Pohozaev identity with the method in [7] obtains the bounded PS sequence. Therefore, we obtain the second solution which has positive energy.

Let $H^{1}\left(\mathbb{R}^{3}\right)$ be the usual Sobolev space equipped with the inner product and norm

$$
(u, v)=\int_{\mathbb{R}^{3}}[a \nabla u \cdot \nabla v+u v], \quad\|u\|=(u, u)^{1 / 2} .
$$

We denote by $|\cdot|_{s}$ the usual $L^{s}\left(\mathbb{R}^{3}\right)$ norm. Then, we have that $H^{1}\left(\mathbb{R}^{3}\right) \hookrightarrow L^{s}\left(\mathbb{R}^{3}\right)$ continuously for $s \in[2,6]$. Hence, there exists $\gamma_{s}$ such that

$$
|u|_{s} \leqslant \gamma_{s}\|u\|, \quad u \in H^{1}\left(\mathbb{R}^{3}\right) .
$$

Let $H=H_{r}^{1}\left(\mathbb{R}^{3}\right)$ be the subspace of $H^{1}\left(\mathbb{R}^{3}\right)$ containing only the radial functions. Then the imbedding $H \hookrightarrow$ $L^{s}\left(\mathbb{R}^{3}\right)$ is compact for $s \in(2,6)[8$, Corollary 1.26 , page 18$]$.
Let $\mathscr{D}^{1,2}\left(\mathbb{R}^{3}\right)$ be the completion of $C_{0}^{\infty}\left(\mathbb{R}^{3}\right)$ with respect to the norm $\|u\|_{\mathscr{D}^{1,2}}=\left(\int_{\mathbb{R}^{3}}|\nabla u|^{2}\right)^{1 / 2}$.

Define the energy functional $J: H^{1}\left(\mathbb{R}^{3}\right) \rightarrow \mathbb{R}$ by

$$
\begin{gathered}
J(u)=\frac{1}{2} \int_{\mathbb{R}^{3}}\left[a|\nabla u|^{2}+u^{2}\right]+\frac{b}{4}\left(\int_{\mathbb{R}^{3}}|\nabla u|^{2}\right)^{2} \\
-\frac{1}{p+1} \int_{\mathbb{R}^{3}}|u|^{p+1}-\int_{\mathbb{R}^{3}} h u .
\end{gathered}
$$

By $p \in(1,5), h \in L^{2}\left(\mathbb{R}^{3}\right)$, we have $J \in C^{1}\left(H^{1}\left(\mathbb{R}^{3}\right), \mathbb{R}\right)$. And, for any $u, v \in H^{1}\left(\mathbb{R}^{3}\right)$,

$$
\begin{aligned}
\left(J^{\prime}(u), v\right)=\int_{\mathbb{R}^{3}} & {[a \nabla u \cdot \nabla v+u v] } \\
& +b\left(\int_{\mathbb{R}^{3}}|\nabla u|^{2}\right) \int_{\mathbb{R}^{3}} \nabla u \cdot \nabla v \\
& -\int_{\mathbb{R}^{3}}|u|^{p-1} u v-\int_{\mathbb{R}^{3}} h v .
\end{aligned}
$$

Furthermore, by $\left(\mathrm{h}_{1}\right), h(x)=h(|x|)$, the functional $J$ is also a $C^{1}$ functional defined on $H$. By standard argument, the weak solution of (1) is corresponding to the critical point of the functional $J$ on $H$.

Our main result is as follows.

Theorem 1. Let $p \in(1,5)$ and h satisfy $\left(\mathrm{h}_{1}\right)-\left(\mathrm{h}_{2}\right)$. Then, problem (1) has at least two nontrivial radial solutions $u_{0}$ and $v_{0}$, satisfying $J\left(u_{0}\right)<0<J\left(v_{0}\right)$.

The paper is organized as follows. In Section 2, we give the existence of the negative energy solution $u_{0}$. The existence of positive energy solution $v_{0}$ and the proof of Theorem 1 are given in Section 3.

\section{Existence of Negative Energy Solution}

In this section, we give the existence of the negative energy solution. In order to obtain our first solution, we need the following preliminaries.

Lemma 2. Let $p \in(1,5)$ and $h$ satisfy $\left(\mathrm{h}_{1}\right)$. Then, there exists $\rho, \alpha>0$ such that $\left.J\right|_{\partial B_{\rho}} \geqslant \alpha$, where $B_{\rho}=\{u \in H: \|$ $u \|<\rho\}$.

Proof. For $u \in H$, by (7), the Hölder inequality and the Sobolev inequality imply that

$$
\begin{aligned}
J(u) \geqslant & \frac{1}{2}\|u\|^{2}-\frac{1}{p+1} \gamma_{p+1}^{p+1}\|u\|^{p+1} \\
& -|h|_{2}|u|_{2} \\
\geqslant & \|u\|\left(\frac{1}{2}\|u\|-\frac{1}{p+1} \gamma_{p+1}^{p+1}\|u\|^{p}-\gamma_{2}|h|_{2}\right) .
\end{aligned}
$$

Set

$$
g(t)=\frac{1}{2} t-\frac{1}{p+1} \gamma_{p+1}^{p+1} t^{p}, \quad t \geqslant 0
$$


since $p>1$, by calculating directly, we see that $\max _{t \geqslant 0} g(t)=$ $g(\rho)>0$, where $\rho=\left((p+1) / 2 p \gamma_{p+1}^{p+1}\right)^{1 /(p-1)}, g(\rho)=((p-$ 1)/2p) $\rho$. Then it follows that, if $|h|_{2} \gamma_{2}<g(\rho)$, that is, $|h|_{2}<$ $\gamma_{2}^{-1} g(\rho) \triangleq m_{p}$, there exists $\alpha=\rho\left(g(\rho)-\gamma_{2}|h|_{2}\right)>0$ such that $\left.J\right|_{\partial B_{\rho}} \geqslant \alpha$, where

$$
m_{p}=\frac{p-1}{2 p \gamma_{2}}\left(\frac{p+1}{2 p \gamma_{p+1}^{p+1}}\right)^{1 /(p-1)} .
$$

Lemma 3. Let $p \in(1,5)$ and $h$ satisfy $\left(\mathrm{h}_{1}\right)$. Then $c=$ $\inf _{\bar{B}_{\rho}} J<0$, where $\rho$ is given by Lemma 2 and $B_{\rho}=\{u \in H$ : $\|u\|<\rho\}$.

Proof. By $\left(\mathrm{h}_{1}\right), h \in L^{2}\left(\mathbb{R}^{3}\right), h \neq 0$, then for $\varepsilon \in\left(0,|h|_{2}\right)$, there exists $\psi \in C_{0}^{\infty}\left(\mathbb{R}^{3}\right)$ such that $|h-\psi|_{2}<\varepsilon$. Then $\int_{\mathbb{R}^{3}}\left(h^{2}-\right.$ $h \psi) \leqslant \int_{\mathbb{R}^{3}}\left|h^{2}-h \psi\right| \leqslant \varepsilon|h|_{2}$, so $\int_{\mathbb{R}^{3}} h \psi \geqslant|h|_{2}^{2}-\varepsilon|h|_{2}>0$. Hence, by (7), for $t>0$ small enough, we have

$$
\begin{aligned}
J(t \psi)= & \frac{t^{2}}{2}\|\psi\|^{2}+\frac{b t^{4}}{4}\left(\int_{\mathbb{R}^{3}}|\nabla \psi|^{2}\right)^{2} \\
& -\frac{t^{p+1}}{p+1} \int_{\mathbb{R}^{3}}|\psi|^{p+1}-t \int_{\mathbb{R}^{3}} h \psi<0 .
\end{aligned}
$$

Then, by the definition of $B_{\rho}, c=\inf _{\bar{B}_{\rho}} J<0$.

Lemma 4. Let $p \in(1,5)$ and $h$ satisfy $\left(\mathrm{h}_{1}\right)$. The bounded PS sequence of the functional J possesses a convergent subsequence.

Proof. Let $\left\{u_{n}\right\}$ be a bounded PS sequence of $J$, that is $\left\{u_{n}\right\}$ and $\left\{J\left(u_{n}\right)\right\}$ are bounded, $J^{\prime}\left(u_{n}\right) \rightarrow 0$ in $H^{\prime}$, where $H^{\prime}$ is the dual space of $H$. We may assume that, up to a subsequence,

$$
\begin{gathered}
u_{n} \rightarrow u \quad \text { in } H, \quad u_{n} \longrightarrow u \quad \text { in } L^{p+1}\left(\mathbb{R}^{3}\right), \\
u_{n} \longrightarrow u \quad \text { a.e. on } \mathbb{R}^{3} .
\end{gathered}
$$

It follows that

$$
\int_{\mathbb{R}^{3}}\left(\left|u_{n}\right|^{p-1} u_{n}-|u|^{p-1} u\right)\left(u_{n}-u\right) \longrightarrow 0, \quad n \longrightarrow \infty .
$$

By (8), we can obtain that

$$
\begin{aligned}
\left(J^{\prime}\left(u_{n}\right)-J^{\prime}(u), u_{n}-u\right) & \\
= & \left\|u_{n}-u\right\|^{2}+b\left(\int_{\mathbb{R}^{3}}\left|\nabla u_{n}\right|^{2}\right) \int_{\mathbb{R}^{3}} \nabla u_{n} \cdot\left(\nabla u_{n}-\nabla u\right) \\
& -b\left(\int_{\mathbb{R}^{3}}|\nabla u|^{2}\right) \int_{\mathbb{R}^{3}} \nabla u \cdot\left(\nabla u_{n}-\nabla u\right) \\
& -\int_{\mathbb{R}^{3}}\left(\left|u_{n}\right|^{p-1} u_{n}-|u|^{p-1} u\right)\left(u_{n}-u\right) \\
= & \left\|u_{n}-u\right\|^{2}+b\left(\int_{\mathbb{R}^{3}}\left|\nabla u_{n}\right|^{2}\right) \int_{\mathbb{R}^{3}}\left|\nabla u_{n}-\nabla u\right|^{2}
\end{aligned}
$$

$$
\begin{aligned}
& +b\left(\int_{\mathbb{R}^{3}}\left(\left|\nabla u_{n}\right|^{2}-|\nabla u|^{2}\right)\right) \int_{\mathbb{R}^{3}} \nabla u \cdot\left(\nabla u_{n}-\nabla u\right) \\
& -\int_{\mathbb{R}^{3}}\left(\left|u_{n}\right|^{p-1} u_{n}-|u|^{p-1} u\right)\left(u_{n}-u\right) .
\end{aligned}
$$

Since $\left\{u_{n}\right\}$ is also bounded in $\mathscr{D}^{1,2}\left(\mathbb{R}^{3}\right)$, then

$$
b\left(\int_{\mathbb{R}^{3}}\left(\left|\nabla u_{n}\right|^{2}-|\nabla u|^{2}\right)\right) \int_{\mathbb{R}^{3}} \nabla u \cdot\left(\nabla u_{n}-\nabla u\right) \longrightarrow 0 .
$$

Therefore,

$$
\begin{aligned}
0 \leqslant & \left\|u_{n}-u\right\|^{2} \leqslant\left\|u_{n}-u\right\|^{2} \\
& +b\left(\int_{\mathbb{R}^{3}}\left|\nabla u_{n}\right|^{2}\right) \int_{\mathbb{R}^{3}}\left|\nabla u_{n}-\nabla u\right|^{2} \longrightarrow 0, \quad n \longrightarrow \infty .
\end{aligned}
$$

That is, $u_{n} \rightarrow u$ in $H$.

Theorem 5. Let $p \in(1,5)$ and $h$ satisfy $\left(\mathrm{h}_{1}\right)$. Then, there exists $u_{0} \in H$ such that

$$
J\left(u_{0}\right)=c=\inf _{\bar{B}_{\rho}} J(u)<0,
$$

where $\rho$ is given by Lemma 2 and $B_{\rho}=\{u \in H:\|u\|<\rho\}$.

Proof. By Lemma 3, $c=\inf \left\{J(u): u \in \bar{B}_{\rho}\right\}<0$, then by Ekeland variational principle [9], there exists $\left\{u_{n}\right\} \subset \bar{B}_{\rho}$ such that

$$
\begin{aligned}
c & \leqslant J\left(u_{n}\right) \leqslant c+\frac{1}{n}, \\
J(\omega) & \geqslant J\left(u_{n}\right)-\frac{1}{n}\left\|\omega-u_{n}\right\| \quad \forall \omega \in \bar{B}_{\rho} .
\end{aligned}
$$

Then, by Lemma $2 u_{n} \in B_{\rho}$, then $\left\{u_{n}\right\}$ is a bounded PS sequence of $J$. Therefore, Lemma 4 implies that there exists $u_{0} \in H$ such that $u_{n} \rightarrow u_{0}$, up to a subsequence. So $J\left(u_{0}\right)=c<0$ and $J^{\prime}\left(u_{0}\right)=0$.

\section{Proof of Theorem 1}

In this section, we will show the existence of the second solution. Note that $p \in(1,5)$, when $p \in(1,3],|u|^{p-1} u$ neither satisfies (AR) condition nor is 4-superlinear. So, in order to obtain the bounded PS sequence, following the argument in [6], we also use a direct method in [7]. Firstly, we recall the following main result in [7]. The "monotonicity trick" at the core of this theorem was invented by Struwe (see [9]).

Theorem 6 (see [7]). Let $(X,\|\cdot\|)$ be a Banach space and $I \subset \mathbb{R}_{+}$be an interval. Consider the family of $C^{1}$ functionals on $X$

$$
J_{\lambda}(u)=A(u)-\lambda B(u), \quad \lambda \in I,
$$


with $B$ nonnegative and either $A(u) \rightarrow \infty$ or $B(u) \rightarrow \infty$ as $\|u\| \rightarrow \infty$. We assume that there are two points $v_{1}$, $v_{2}$ in $X$ such that

$$
c_{\lambda}=\inf _{\gamma \in \Gamma_{\lambda}} \max _{t \in[0,1]} J_{\lambda}(\gamma(t))>\max \left\{J_{\lambda}\left(v_{1}\right), J_{\lambda}\left(v_{2}\right)\right\}, \quad \forall \lambda \in J,
$$

where

$$
\Gamma=\left\{\gamma \in C([0,1], X): \gamma(0)=v_{1}, \gamma(1)=v_{2}\right\} .
$$

Then, for almost every $\lambda \in I$ there is a sequence $\left\{u_{n}(\lambda)\right\} \subset$ $X$ such that

(i) $\left\{u_{n}(\lambda)\right\}$ is bounded;

(ii) $J_{\lambda}\left(u_{n}(\lambda)\right) \rightarrow c_{\lambda}$;

(iii) $J_{\lambda}^{\prime}\left(u_{n}(\lambda)\right) \rightarrow 0$ in the dual $X^{-1}$ of $X$. $\mathbb{R}$ by

In our case, $X=H, I=[1 / 2,1]$, and define $J_{\lambda}: X \rightarrow$

$$
J_{\lambda}(u)=A(u)-\lambda B(u),
$$

where $\lambda \in I$,

$$
\begin{aligned}
A(u)= & \frac{1}{2}\|u\|^{2}+\frac{b}{4}\left(\int_{\mathbb{R}^{3}}|\nabla u|^{2}\right)^{2} \\
& -\int_{\mathbb{R}^{3}} h u, B(u)=\frac{1}{p+1} \int_{\mathbb{R}^{3}}|u|^{p+1} .
\end{aligned}
$$

Then $\left\{J_{\lambda}\right\}_{\lambda \in I}$ is a family of $C^{1}$ functionals on $H$. For any $u \in$ $H, B(u) \geqslant 0$, and $A(u) \geqslant(1 / 2)\|u\|^{2}-\gamma_{2}|h|_{2}\|u\| \rightarrow$ $\infty$ as $\|u\| \rightarrow \infty$.

In the following, we verify that the functional $J_{\lambda}$ satisfies the conditions of Theorem 6 .

Lemma 7. Let $p \in(1,5)$ and $h$ satisfy $\left(\mathrm{h}_{1}\right)-\left(\mathrm{h}_{2}\right)$. Then, the following claims hold:

(i) there exist $r, a>0$ and $e \in H$ such that for all $\lambda \in I$

$$
\left.J_{\lambda}\right|_{\partial B_{\rho}}(u) \geqslant a>0, \quad J_{\lambda}(e)<0 \quad \text { with }\|e\|>r ;
$$

(ii) for any $\lambda \in I$,

$$
c_{\lambda}=\inf _{\gamma \in \Gamma} \max _{t \in[0,1]} J_{\lambda}(\gamma(t))>\max \left\{J_{\lambda}(0), J_{\lambda}(e)\right\},
$$

where

$$
\Gamma=\{\gamma \in C([0,1], X): \gamma(0)=0, \gamma(1)=e\} .
$$

Proof. (i) Since for all $u \in H$ and $\lambda \in I=[1 / 2,1], J_{\lambda}(u) \geqslant$ $J_{1}(u)$. By Lemma 2, there exist $r, a>0$ independent of $\lambda \in$ $I$ such that $J_{\lambda}(u) \geqslant a>0$ with $\|u\|=r$.

We choose a function $w \in H$ and $w \neq 0$. Set $w_{t}(\cdot)=$ $t w\left(t^{-2} \cdot\right)$ for $t>0$. Then, for all $\lambda \in I$, by (7) and $\left(\mathrm{h}_{1}\right)$, we have

$$
\begin{aligned}
J_{\lambda}\left(w_{t}\right) \leqslant \frac{a t^{4}}{2} \int_{\mathbb{R}^{3}}|\nabla w|^{2}+\frac{t^{8}}{2} \int_{\mathbb{R}^{3}}|w|^{2} \\
+\frac{b t^{8}}{4}\left(\int_{\mathbb{R}^{3}}|\nabla w|^{2}\right)^{2}-\frac{t^{p+7}}{2(p+1)} \int_{\mathbb{R}^{3}}|w|^{p+1}
\end{aligned}
$$

Noting $p>1$, then there exists $t_{0}$ large enough satisfying $\left\|w_{t_{0}}\right\|>r$, which is independent of $\lambda \in I$, such that for all $\lambda \in I, J_{\lambda}(e)<0$ with $e=w_{t_{0}}$.

(ii) Since $c_{\lambda}$ is nonincreasing on $\lambda \in I$, then by the definition of $c_{\lambda}$ and (i), for all $\lambda \in I$, we have $c_{1 / 2} \geqslant c_{\lambda} \geqslant$ $c_{1} \geqslant a>0$.

By Lemma 7 and Theorem 6, for almost every $\lambda \in I$, there exists a bounded sequence $\left\{u_{n}^{\lambda}\right\} \subset H$ such that $J_{\lambda}\left(u_{n}^{\lambda}\right) \rightarrow$ $c_{\lambda},\left(J_{\lambda}\right)^{\prime}\left(u_{n}^{\lambda}\right) \rightarrow 0$. By Lemma 4 , there exists $u^{\lambda} \in H$ such that $u_{n}^{\lambda} \rightarrow u^{\lambda}$ in $H$. Therefore, $\left(J_{\lambda}\right)^{\prime}\left(u^{\lambda}\right)=0$ and $J_{\lambda}\left(u^{\lambda}\right)=$ $c_{\lambda}$. It follows from (ii) of Lemma 7 that $u^{\lambda} \neq 0$.

Therefore, there exists $\left\{\lambda_{n}\right\} \subset I$ with $\lambda_{n} \rightarrow 1^{-}$and a nonnegative sequence $\left\{u^{\lambda_{n}}\right\}$ (denoted by $\left\{u_{n}\right\}$ for simplicity) satisfying

$$
J_{\lambda_{n}}\left(u_{n}\right)=c_{\lambda_{n}}, \quad\left(J_{\lambda_{n}}\right)^{\prime}\left(u_{n}\right)=0
$$

In order to obtain the boundedness of $\left\{u_{n}\right\}$, we need the following Pohozaev identity. The proof is similar to the argument in [10].

Lemma 8. Under the conditions of $\left(\mathrm{h}_{1}\right)$ and $\left(\mathrm{h}_{2}\right)$, if $u \in H$ is a weak solution of (1), the following Pohozaev identity holds:

$$
\begin{aligned}
\frac{1}{2}(a & \left.+b \int_{\mathbb{R}^{3}}|\nabla u|^{2}\right) \int_{\mathbb{R}^{3}}|\nabla u|^{2}+\frac{3}{2} \int_{\mathbb{R}^{3}} u^{2} \\
& =\frac{3}{p+1} \int_{\mathbb{R}^{3}}|u|^{p+1}+\int_{\mathbb{R}^{3}}(3 h+(\nabla h(x), x)) u .
\end{aligned}
$$

Lemma 9. Consider $\left\{u_{n}\right\}$ in (29) is bounded in $H$.

Proof. Firstly, since $\left(J_{\lambda_{n}}\right)^{\prime}\left(u_{n}\right)=0$, then by Lemma $8, u_{n}$ satisfies the following Pohozaev identity:

$$
\begin{gathered}
\frac{a}{2} \int_{\mathbb{R}^{3}}\left|\nabla u_{n}\right|^{2}+\frac{b}{2}\left(\int_{\mathbb{R}^{3}}\left|\nabla u_{n}\right|^{2}\right)^{2}+\frac{3}{2} \int_{\mathbb{R}^{3}} u_{n}^{2} \\
-\lambda_{n} \frac{3}{p+1} \int_{\mathbb{R}^{3}}\left|u_{n}\right|^{p+1}-3 \int_{\mathbb{R}^{3}} h(x) u_{n} \\
-\int_{\mathbb{R}^{3}}(\nabla h(x), x) u_{n}=0 .
\end{gathered}
$$

On the other hand, by $\left(\left(J_{\lambda_{n}}\right)^{\prime}\left(u_{n}\right), u_{n}\right)=0$ and $J_{\lambda_{n}}\left(u_{n}\right)=c_{\lambda_{n}}$, we have that

$$
\begin{gathered}
a \int_{\mathbb{R}^{3}}\left|\nabla u_{n}\right|^{2}+b\left(\int_{\mathbb{R}^{3}}\left|\nabla u_{n}\right|^{2}\right)^{2}+\int_{\mathbb{R}^{3}} u_{n}^{2} \\
-\lambda_{n} \int_{\mathbb{R}^{3}}\left|u_{n}\right|^{p+1}-\int_{\mathbb{R}^{3}} h u_{n}=0, \\
\frac{a}{2} \int_{\mathbb{R}^{3}}\left|\nabla u_{n}\right|^{2}+\frac{b}{4}\left(\int_{\mathbb{R}^{3}}\left|\nabla u_{n}\right|^{2}\right)^{2}+\frac{1}{2} \int_{\mathbb{R}^{3}} u_{n}^{2} \\
\quad-\frac{\lambda_{n}}{p+1} \int_{\mathbb{R}^{3}}\left|u_{\mathrm{n}}\right|^{p+1}-\int_{\mathbb{R}^{3}} h u_{n}=c_{\lambda_{n}} .
\end{gathered}
$$


Then, combining (33) with (31), we can obtain

$$
a \int_{\mathbb{R}^{3}}\left|\nabla u_{n}\right|^{2}+\frac{b}{4}\left(\int_{\mathbb{R}^{3}}\left|\nabla u_{n}\right|^{2}\right)^{2}=3 c_{\lambda_{n}}-\int_{\mathbb{R}^{3}}(\nabla h(x), x) u_{n} .
$$

Since $c_{\lambda_{n}} \leqslant c_{1 / 2}$ by Lemma 7 , and $(\nabla h(x), x) \in L^{2}\left(\mathbb{R}^{3}\right)$, so in order to prove the boundedness of $\left\{u_{n}\right\}$ in $H$, we only need to prove that $\left|u_{n}\right|_{2}$ is bounded. By contradiction, we assume that $\left|u_{n}\right|_{2} \rightarrow \infty$, up to a subsequence. Let $v_{n}=$ $u_{n} /\left|u_{n}\right|_{2}, X_{n}=a \int_{\mathbb{R}^{3}}\left|\nabla v_{n}\right|^{2}, Y_{n}=b\left|u_{n}\right|_{2}^{2}\left(\int_{\mathbb{R}^{3}}\left|\nabla v_{n}\right|^{2}\right)^{2}, Z_{n}=$ $\lambda_{n}\left|u_{n}\right|_{2}^{p-1} \int_{\mathbb{R}^{3}}\left|v_{n}\right|^{p+1}$.

Note that $c_{\lambda_{n}}$ is bounded and $h,(\nabla h(x), x) \in L^{2}\left(\mathbb{R}^{3}\right)$. Multiplying (31)-(33) by $1 /\left|u_{n}\right|_{2}^{2}$, we have that

$$
\begin{gathered}
\frac{X_{n}}{2}+\frac{Y_{n}}{2}-\frac{3}{p+1} Z_{n}=-\frac{3}{2}+o(1), \\
X_{n}+Y_{n}-Z_{n}=-1+o(1), \\
\frac{X_{n}}{2}+\frac{Y_{n}}{4}-\frac{Z_{n}}{p+1}=-\frac{1}{2}+o(1),
\end{gathered}
$$

where $o(1)$ denotes the quantity tends to zero as $n \rightarrow \infty$. By calculating, we obtain that

$$
X_{n}=\frac{1-p}{5-p}+o(1) .
$$

Since $p \in(1,5)$ and $X_{n} \geqslant 0$ for $n \in \mathbb{N}$, so this is a contradiction for $n$ large enough. Therefore, $\left\{u_{n}\right\}$ is bounded in $H$.

Proof of Theorem 1. Since

$$
\begin{aligned}
J\left(u_{n}\right)=J_{\lambda_{n}} & \left(u_{n}\right)+\left(\lambda_{n}-1\right) \frac{1}{p+1} \int_{\mathbb{R}^{3}}\left|u_{n}\right|^{p+1}, \\
\left(J^{\prime}\left(u_{n}\right), v\right)= & \left(\left(J_{\lambda_{n}}\right)^{\prime}\left(u_{n}\right), v\right) \\
& +\left(\lambda_{n}-1\right) \int_{\mathbb{R}^{3}}\left|u_{n}\right|^{p-1} u_{n} v, \quad v \in H .
\end{aligned}
$$

By Lemma 9 and $H \hookrightarrow L^{p+1}\left(\mathbb{R}^{3}\right),\left|u_{n}\right|_{p+1}$ is bounded and $\left.\left.\left|\int_{\mathbb{R}^{3}}\right| u_{n}\right|^{p-1} u_{n} v\left|\leqslant \gamma_{p+1}^{p+1}\right| u_{n}\right|_{p+1} ^{p}\|v\|$. Thus, when $\lambda_{n} \rightarrow$ $1^{-}$, we have that $\left\{J\left(u_{n}\right)\right\}$ is bounded and $J^{\prime}\left(u_{n}\right) \rightarrow 0$. Therefore $\left\{u_{n}\right\}$ is a bounded PS sequence of $J$. By Lemma 4 , $\left\{u_{n}\right\}$ has a convergent subsequence. We may assume that $u_{n} \rightarrow v_{0}$, up to a subsequence. Consequently, $J^{\prime}\left(v_{0}\right)=0$. According to Lemma 7, we have $J\left(v_{0}\right)=\lim _{n \rightarrow \infty} J\left(u_{n}\right)=$ $\lim _{n \rightarrow \infty} J_{\lambda_{n}}\left(u_{n}\right) \geqslant a>0$. Thus $v_{0}$ is a positive energy solution to problem (1). Hence, by Theorem 5 problem (1) has two solutions $u_{0}$ and $v_{0}$ satisfying $J\left(u_{0}\right)<0<J\left(v_{0}\right)$.

\section{Acknowledgments}

The authors thank the anonymous referee for the careful reading and some helpful comments, which greatly improve the paper. Projects supported by the National Natural Science Foundation of China (Grant no. 11071149), Science Council of Shanxi Province (2010011001-1, 2012011004-2, 2013021001-4).

\section{References}

[1] X. He and W. Zou, "Existence and concentration behavior of positive solutions for a Kirchhoff equation in $R^{3}$," Journal of Differential Equations, vol. 252, no. 2, pp. 1813-1834, 2012.

[2] J. Jin and X. Wu, "Infinitely many radial solutions for Kirchhofftype problems in $R^{N}$," Journal of Mathematical Analysis and Applications, vol. 369, no. 2, pp. 564-574, 2010.

[3] W. Liu and X. He, "Multiplicity of high energy solutions for superlinear Kirchhoff equations," Journal of Applied Mathematics and Computing, vol. 39, no. 1-2, pp. 473-487, 2012.

[4] J. Wang, L. Tian, J. Xu, and F. Zhang, "Multiplicity and concentration of positive solutions for a Kirchhoff type problem with critical growth," Journal of Differential Equations, vol. 253, no. 7, pp. 2314-2351, 2012.

[5] X. Wu, "Existence of nontrivial solutions and high energy solutions for Schrödinger-Kirchhoff-type equations in $\mathbf{R}^{N}$," Nonlinear Analysis, vol. 12, no. 2, pp. 1278-1287, 2011.

[6] Y. Li, F. Li, and J. Shi, "Existence of a positive solution to Kirchhoff type problems without compactness conditions," Journal of Differential Equations, vol. 253, no. 7, pp. 2285-2294, 2012.

[7] L. Jeanjean, "On the existence of bounded Palais-Smale sequences and application to a Landesman-Lazer-type problem set on $\mathbf{R}^{N}$," Proceedings of the Royal Society of Edinburgh A, vol. 129, no. 4, pp. 787-809, 1999.

[8] M. Willem, Minimax Theorems, Progress in Nonlinear Differential Equations and their Applications, 24, Birkhäuser, Boston, Mass, USA, 1996.

[9] M. Struwe, Variational Methods, Applications to Nonlinear Partial Differential Equations and Hamiltonian Systems, Springer, Berlin, Germany, 1990.

[10] H. Berestycki and P.-L. Lions, "Nonlinear scalar field equations. I. Existence of a ground state," Archive for Rational Mechanics and Analysis, vol. 82, no. 4, pp. 313-345, 1983. 


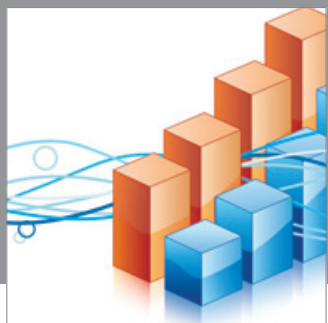

Advances in

Operations Research

mansans

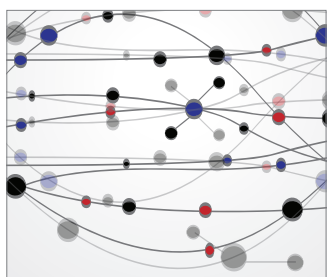

The Scientific World Journal
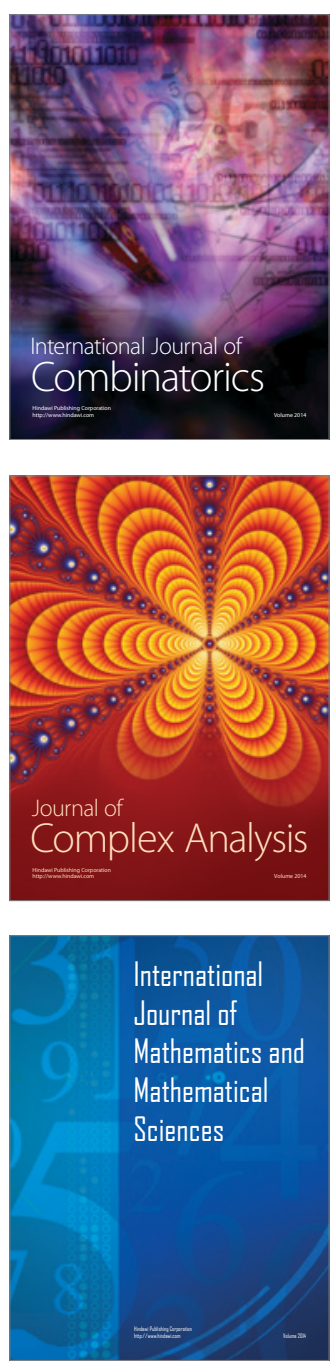
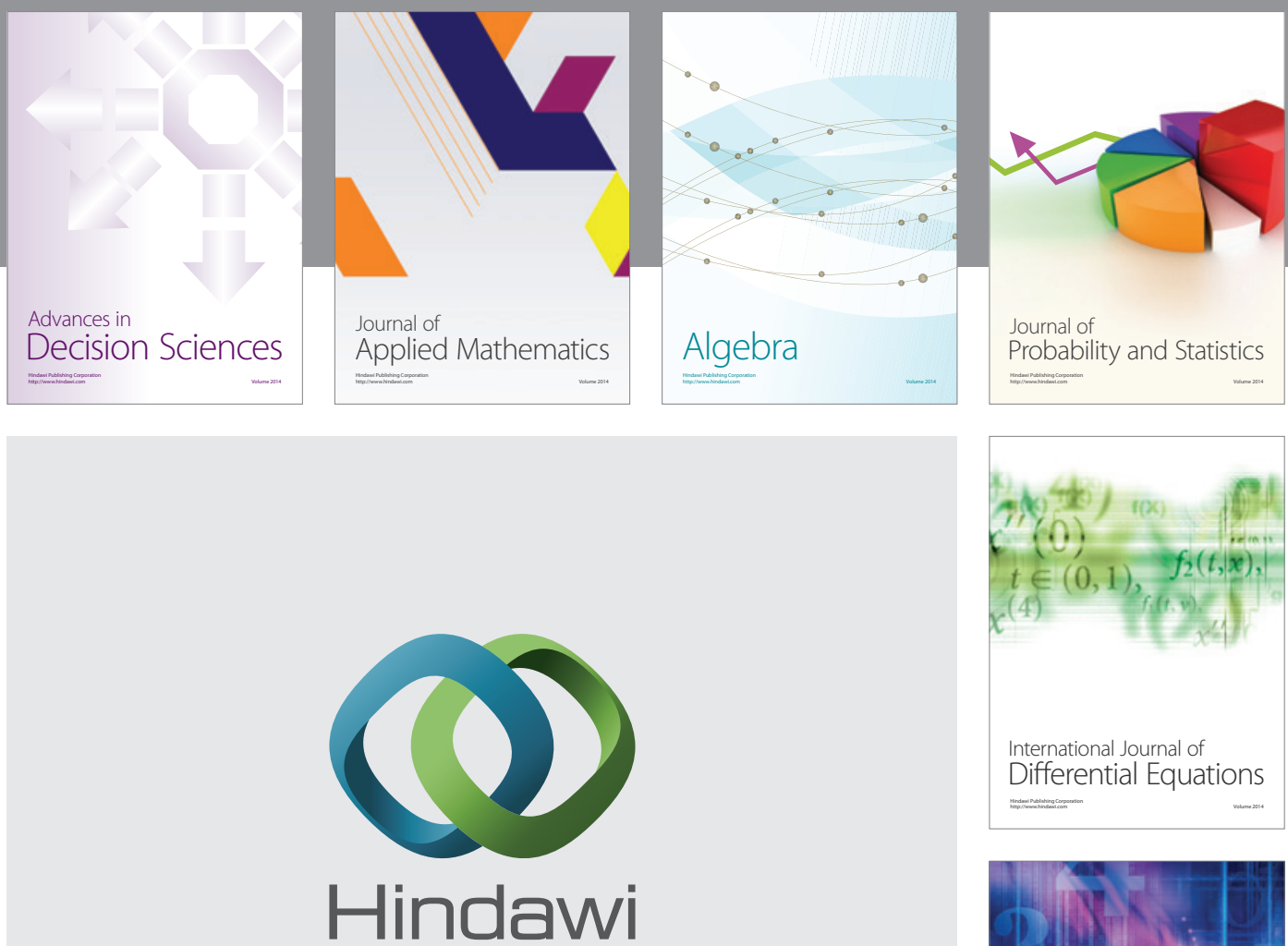

Submit your manuscripts at http://www.hindawi.com
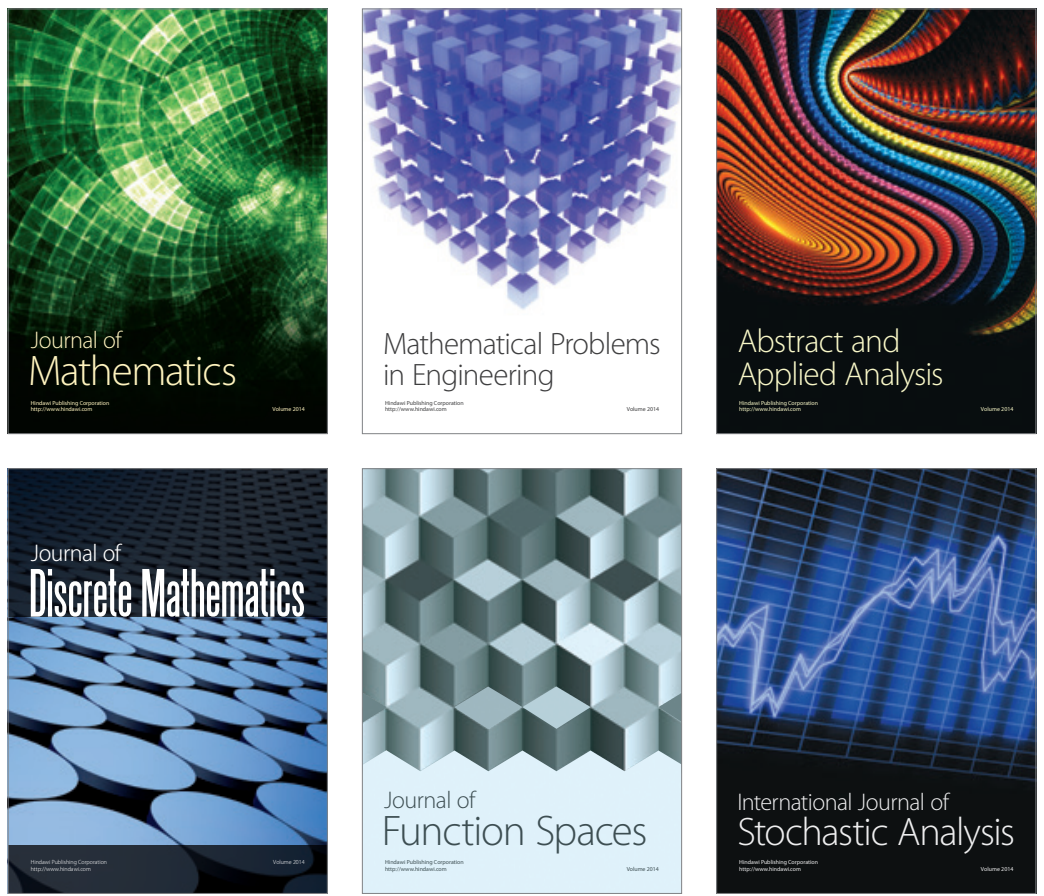

Journal of

Function Spaces

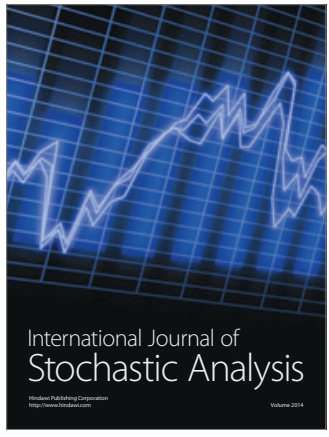

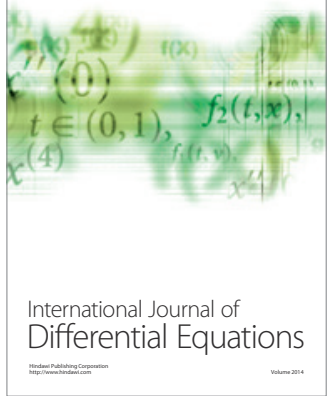
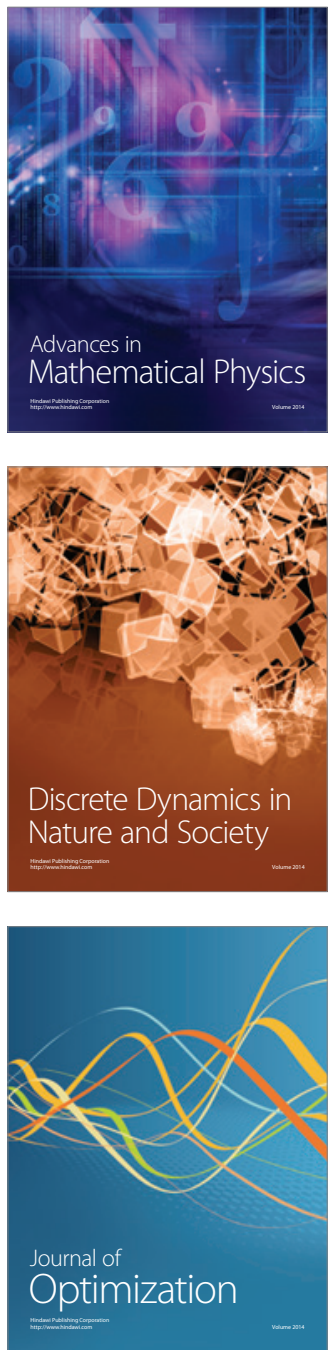